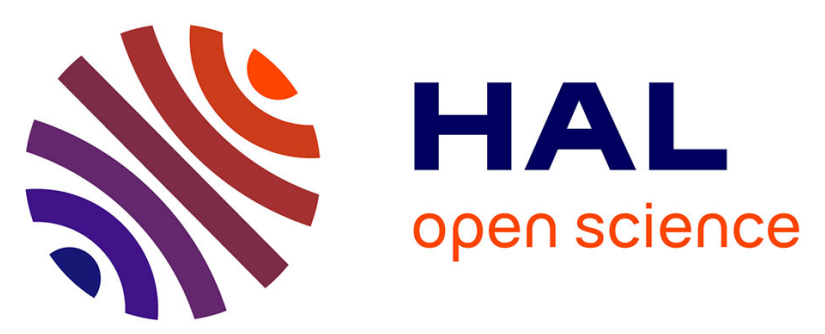

\title{
Influence of Carbene and Phosphine Ligands on the Catalytic Activity of Gold Complexes in the Hydroamination and Hydrohydrazination of Alkynes
}

Sima Yazdani, Glen P Junor, Jesse L Peltier, Milan Gembicky, Rodolphe Jazzar, Douglas B Grotjahn, Guy Bertrand

\section{To cite this version:}

Sima Yazdani, Glen P Junor, Jesse L Peltier, Milan Gembicky, Rodolphe Jazzar, et al.. Influence of Carbene and Phosphine Ligands on the Catalytic Activity of Gold Complexes in the Hydroamination and Hydrohydrazination of Alkynes. ACS Catalysis, 2020, 10 (9), pp.5190-5201. 10.1021/acscatal.0c01352 . hal-03083287

\section{HAL Id: hal-03083287 https://hal.science/hal-03083287}

Submitted on 18 Dec 2020

HAL is a multi-disciplinary open access archive for the deposit and dissemination of scientific research documents, whether they are published or not. The documents may come from teaching and research institutions in France or abroad, or from public or private research centers.
L'archive ouverte pluridisciplinaire HAL, est destinée au dépôt et à la diffusion de documents scientifiques de niveau recherche, publiés ou non, émanant des établissements d'enseignement et de recherche français ou étrangers, des laboratoires publics ou privés. 


\title{
Influence of Carbene and Phosphine Ligands on the Catalytic Activ- ity of Gold Complexes in the Hydroamination and Hydrohydrazi- nation of Alkynes
}

\author{
Sima Yazdani, ${ }^{\dagger}, \ddagger$ Glen P. Junor, ${ }^{\dagger}$ Jesse L. Peltier, ${ }^{\dagger}$ Milan Gembicky, ${ }^{\dagger}$ Rodolphe Jazzar, ${ }^{*}$, Douglas B. \\ Grotjahn,*,‡ Guy Bertrand*,† \\ + UCSD-CNRS Joint Research Laboratory (UMI 3555), Department of Chemistry and Biochemistry, University of Califor- \\ nia, San Diego, La Jolla, California 92093-0358, United States \\ * Department of Chemistry and Biochemistry, San Diego State University, 5500 Campanile Drive, San Diego, California \\ 92182-1030, United States
}

Supporting Information Placeholder

\begin{abstract}
Through kinetic measurements, catalytic and stoichiometric experiments, as well as DFT calculations, we compare the catalytic activity of gold complexes supported by a variety of ligands $\left(\mathrm{R}_{3} \mathrm{P}, \mathrm{NHC}\right.$, CAAC-5, CAAC-6, BiCAAC) in the hydroamination and hydrohydrazination of alkynes. This study provides a rationale for the superior efficiency of a gold complex bearing a bicyclic (alkyl)(amino)carbene (BiCAAC). We demonstrate that this ligand motif, which is readily available, provides a durable gold catalyst able to compete with sophisticated state-of-the-art phosphine and NHC ligands, which feature secondary interaction capabilities.
\end{abstract}

KEYWORDS: cyclic (alkyl)(amino) carbene, gold catalysis, hydroamination, hydrohydrazination, mechanistic study

The hydroamination of carbon-carbon multiple bonds is the most atom-efficient method for the introduction of nitrogen fragments into organic molecules. ${ }^{1}$ Pioneered by Tanaka et al. ${ }^{2}$ using $\left(\mathrm{PPh}_{3}\right) \mathrm{AuNTf}_{2}$, the field of coinage metal hydroamination catalysis has witnessed an outstanding development in recent years. ${ }^{3}$ $\mathrm{Xu}$ et al. ${ }^{4}$ observed a remarkable ligand effect ${ }^{5}$ in the intermolecular hydroamination of phenyl acetylene with aniline, using a set of phosphine ligands. Sophisticated phosphines, ${ }^{6,7}$ such as the WangPhos $\mathbf{L}_{\mathbf{A}}{ }^{8}$ or the Lavallo anionic phosphine $\mathbf{L}_{\mathbf{B}},{ }^{9}$ allowed to achieve up to 22,000 TON for the hydroamination of phenyl acetylene with aniline. More recently, NHC ligands have become popular replacements for phosphines. As an illustration, César, Michelet and co-workers reported that a gold complex bearing $\mathrm{NHC} \mathbf{L}_{\mathbf{C}},{ }^{10}$ which features a barbituric heterocycle, efficiently promotes the same transformation up to 7600 TON (Scheme 1).
From this short analysis, it appears that phosphines and carbenes, capitalizing on elaborate secondary interactions, afford the most efficient catalysts. However, the presence of these complex motifs clouds an understanding of the electronic contributions from the core of the ligand.11 To better understand, we herein report a detailed investigation of the influence of core ligand structures $\left(\mathrm{R}_{3} \mathrm{P}, \mathrm{NHC}, \mathrm{CAAC}-5, \mathrm{CAAC}-6\right.$, BiCAAC) on the catalytic performance of gold complexes in the hydroamination and hydrohydrazination of alkynes. For a thorough comparison, in-situ kinetic measurements were conducted to determine the order of each reactant and the influence of the electronic properties of the substrates (Hammett plot). Moreover, reactivity studies and DFT calculations enable a

Scheme 1. Comparison of the most efficient tailormade phosphines and carbenes in the hydroamination of phenyl acetylene with aniline.

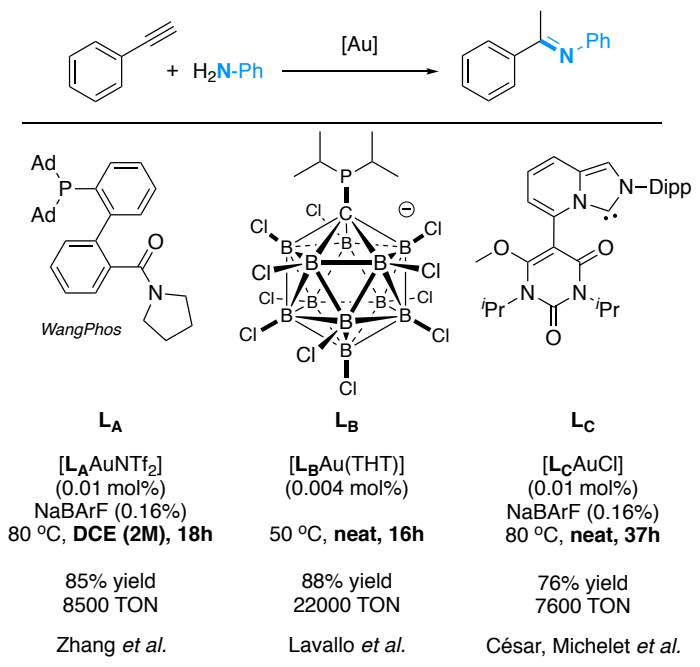


rationalization of the catalytic performance of each ligand system. Finally, we show that the readily accessible BiCAAC, ${ }^{12}$ which does not feature secondary interactions, competes with state-of-the-art ligands.

\section{Results and Discussion}

We have already shown that gold complexes bearing a 5-membered cyclic (alkyl)(amino) carbene (CAAC-5) 13 are excellent catalysts for the intermolecular hydroamination and hydrohydrazination of alkynes and allenes. ${ }^{14,15,16}$ They survive drastic conditions (up to $\left.200{ }^{\circ} \mathrm{C}\right),{ }^{17}$ which allowed the use of ammonia and parent hydrazine that are difficult substrates for transition metal catalysts. Recently, we extended the family of CAACs to the bicyclic version (BiCAAC) ${ }^{12}$ and the 6membered CAAC (CAAC-6), ${ }^{18}$ which feature distinct electronic and steric properties (Figure 1).

\section{Hydroamination reaction}

Reaction optimization. Inspired by Blackmond et $a l .,{ }^{19}$ we envisaged the use of in-situ kinetic measurements to optimize the catalytic system. Using ${ }^{1} \mathrm{H}$ NMR spectroscopy, we monitored the formation of imine $\mathbf{1 a}$ in the reaction of aniline with para-(methoxyphenyl)

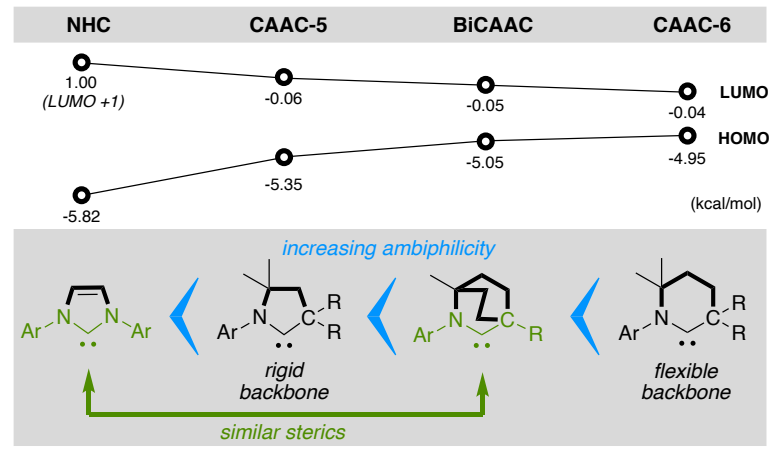

Figure 1. Electronic properties of different families of carbene ligands.

acetylene. The reactions were carried out at room temperature in air, using $\mathrm{KBAr}^{\mathrm{F}}$ as a chloride scavenger, meanwhile changing the nature of the catalyst (Figure 2a-b), the solvent (Figure 2c), the concentration and catalyst loading (Figure $2 \mathrm{~d}$ ). No reaction was observed with (THT)AuCl and using $\left(\mathrm{Ph}_{3} \mathrm{P}\right) \mathrm{AuCl}$, we confirmed the accuracy of our kinetic protocol by obtaining comparable results to those reported by $\mathrm{Xu}$ et al. ${ }^{4 a}$ We found that all the carbene ligands that we considered give rise to more active catalysts than $\mathrm{PPh}_{3}$;
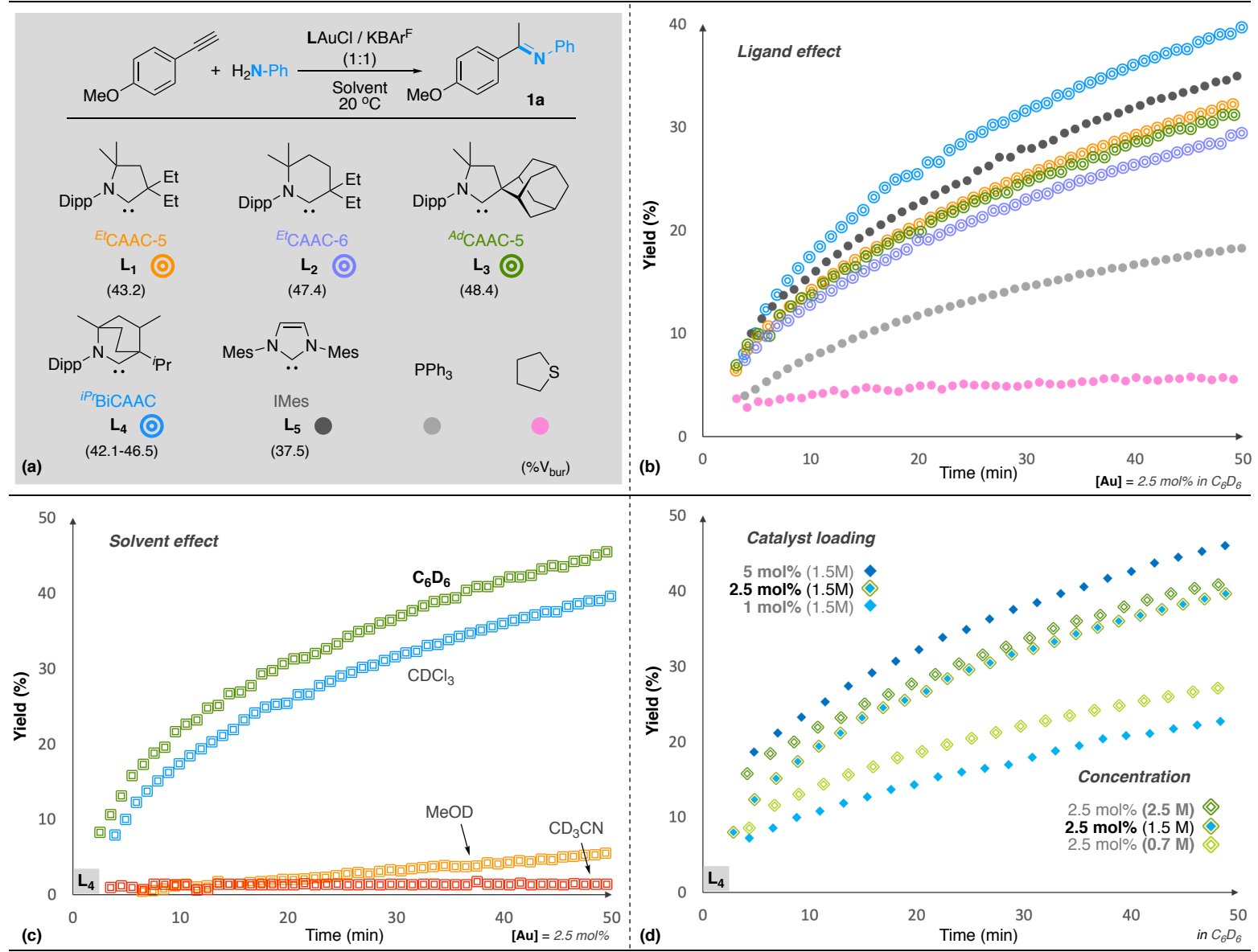

Figure 2. Optimization of the reaction conditions for the hydroamination reaction using ${ }^{1} \mathrm{H}$ NMR kinetic monitoring. 
the order of catalytic activity being BiCAAC $\left(\mathbf{L}_{4}\right)>N$ heterocylic carbene IMes $\left(\mathbf{L}_{5}\right)>E t C A A C-5\left(\mathbf{L}_{1}\right)=A d$ CAAC-5 $\left(\mathbf{L}_{3}\right)>{ }^{E t C A A C}-6\left(\mathbf{L}_{2}\right)$. Comparing the \% buried volumes $\left(\% \mathrm{~V}_{\text {bur }}\right)\left(\mathbf{L}_{4}: 42.1-46.5 ; \mathbf{L}_{5}: 37.5 ; \mathbf{L}_{1}: 43.2\right.$; $\mathbf{L}_{3}: 48.4 ; \mathbf{L}_{2}: 47.4$ ), and the HOMO/LUMO energies of these carbenes ( $\mathbf{L}_{\mathbf{4}}:-5.05 /-0.05 ; \mathbf{L}_{\mathbf{5}}:-5.82 / 1.00 ; \mathbf{L}_{\mathbf{1}}$ and $\mathbf{L}_{3}:-5.35 /-0.06 ; \mathbf{L}_{2}:-4.95 /-0.04 \mathrm{eV}$, it appears that neither the steric hindrance nor the electronic factors are decisive on their own. Using DFT calculations, we realized that the steric demands of BiCAAC $\mathbf{L}_{\mathbf{4}}$ are variable, due to the rotation of the iso-propyl group (Figure 3). Concomitantly, BiCAAC $\mathbf{L}_{\mathbf{4}}$ is both
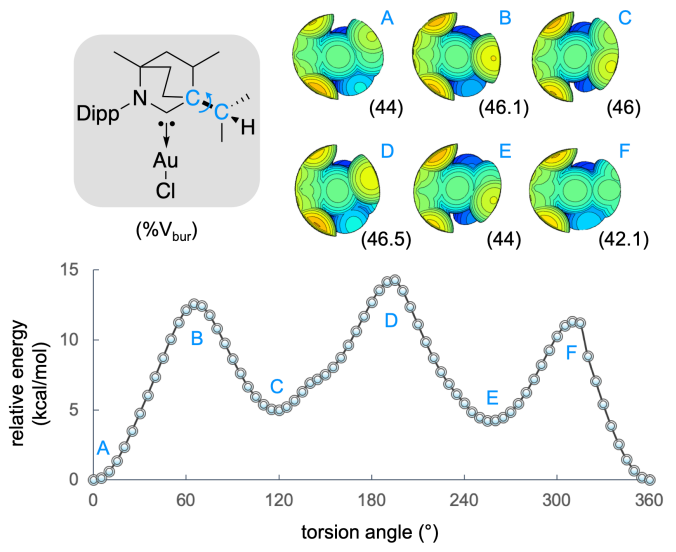

more electron donating and withdrawing than the other carbenes, except for CAAC- $6 \mathbf{L}_{2}$ (Figure 1). Thus, we rationalize the superior performance of IPrBiCAAC $\mathbf{L}_{\mathbf{4}}$ as a compromise between moderate steric bulk, approaching that of $\mathbf{L}_{5}$, and significant ambiphilicity, nearing $\mathbf{L}_{2}$. With respect to the nature of the solvent (Figure 2c), using $\mathbf{L}_{\mathbf{4}}$ we found that the catalytic activity is greatly impaired by strongly coordinating solvents. This is readily rationalized as a competition between the solvent and the substrates for binding to the metal. Finally, we optimized the concentration and the catalyst loading (Figure 2d) and selected $2.5 \mathrm{~mol} \%$ and $1.5 \mathrm{M}$, respectively, as a middle point between conversion and reaction time.

Reaction Scope. Using the optimized catalytic system $\left(2.5 \mathrm{~mol} \%\right.$ of ${ }^{I P r} \mathrm{BiCAACAuCl} / \mathrm{KBAr}^{\mathrm{F}}$ in benzene $1.5 \mathrm{M}$ ), we evaluated the scope of the hydroamination using a variety of alkynes and anilines (Scheme 2). $p$-Toluidine efficiently added to aryl substituted terminal alkynes; all reactions proceeded within a few hours in very high yields at $20{ }^{\circ} \mathrm{C}(\mathbf{1 a - 1 e})$. Alkyl substituted terminal alkynes required higher reaction temperatures to achieve high conversions within a reasonable timeframe $(\mathbf{1 f}-\mathbf{1 g})$. Note that even with these alkynes, full conversion can still be

Figure 3. Illustrating the flexible sterics in ${ }^{I P r} \mathrm{Bi}-$ CAACAuCl.

\section{Scheme 2. Substrate scope with amines and alkynes. NMR yields (isolated yields).}

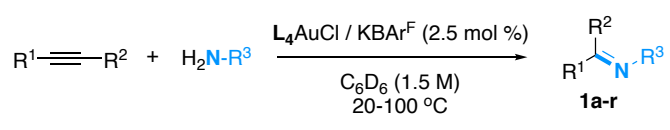
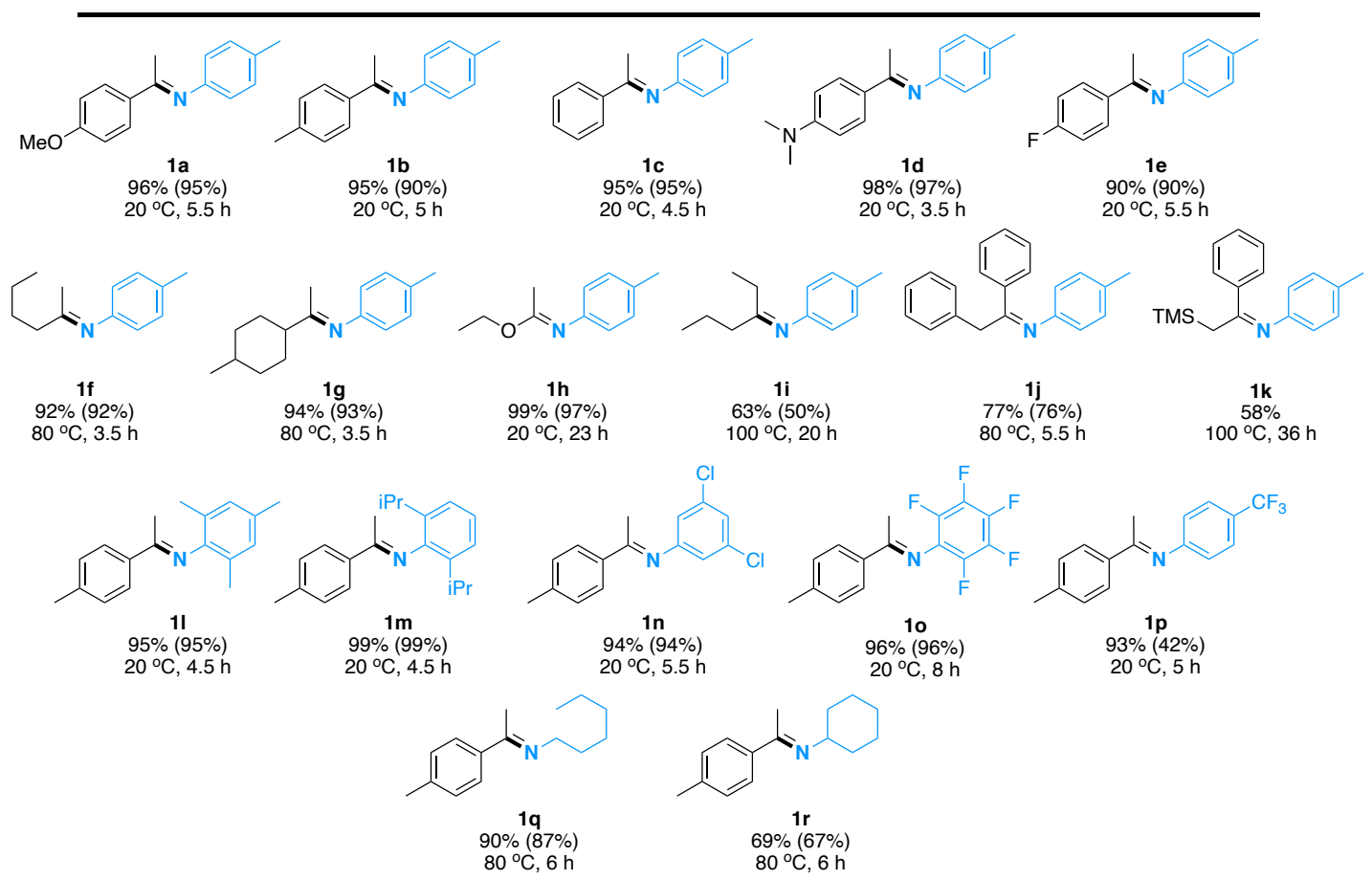
achieved at room temperature albeit with a longer reaction time, which is useful for low boiling point substrates such as ethoxyethyne (1h). We also found internal alkynes to be more challenging (1i-1k) requiring longer reaction times and higher temperatures. We also changed the nature of the aniline (11-1p), and observed very good conversions even with the sterically demanding 2,6-diisopropylaniline (1m) and electron-poor perfluorinated aniline (10). Finally, alkyl amines (1q,r) proved less reactive but were successfully converted to the desired products by performing the reaction at $80^{\circ} \mathrm{C}$.

Hammett correlation study. We conducted a Hammett correlation study to quantify the electronic influence of the substrates (Figure 4). Both anilines and arylalkynes with electron-donating para-substituents showed faster reaction kinetics ( $\rho$ value $<0$ ). Electronrich alkynes favor a forward equilibrium by stabilizing the cationic $\mathrm{Au}-\pi$ complex, whereas electron-rich anilines push a forward equilibrium by favoring the nucleophilic attack on the positively charged carbon. Note, that we observed a nonlinear behavior with the $p$ - $\mathrm{NMe}_{2}$ substituent, likely due to a competitive binding of this group to the gold center.

Rate order. We established the rate order in the alkyne and the amine under pseudo first-order conditions (excess of the spectator substrate; Figure 5). In both cases, we found first order dependence on these substrates which suggests that both of them are involved in the rate-limiting step.

\section{Hydrohydrazination reaction}

Hydrazines are inherently more challenging than amines due to their propensity to generate inert gold(0) nanoparticles ${ }^{20}$ or unreactive Werner complexes. ${ }^{21,22}$ We have shown that (CAAC-5)AuCl in the presence of $\mathrm{KBAr}^{\mathrm{F}}$ is active for the hydrohydrazination of a variety of alkynes at rather high temperatures (90-150 $\left.{ }^{\circ} \mathrm{C}\right) \cdot{ }^{14}$ Although the scope of applications is much narrower, gold complexes bearing the saturated abnormal NHC (saNHC) ${ }^{23}$ or the anti-Bredt-NHC,24,25 which are $\pi$-accepting carbenes, promote this transformation under milder conditions.

Reaction conditions: As we did for amines, we began our investigation with a kinetic survey using ${ }^{1} \mathrm{H}$ NMR spectroscopy to monitor the formation of $2 \mathrm{c}$ in the reaction of phenyl hydrazine with para-methoxy-phenyl acetylene at room temperature. We varied the nature of the catalyst (Figure 6a-b), the solvent (Figure 6c), the concentration and the catalyst loading (Figure 6d). Here again using $\operatorname{KBAr}^{\mathrm{F}}$ as a chloride scavenger we confirmed the accuracy of our kinetic protocol by verifying that $\left(\mathrm{Ph}_{3} \mathrm{P}\right) \mathrm{AuCl}$ is not an efficient catalyst for

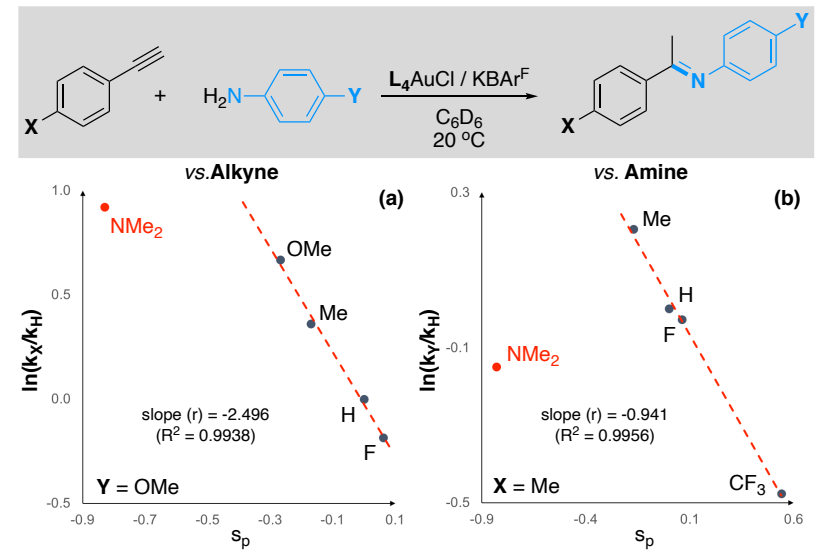

Figure 4. Hammett correlations of rates and substituents on either alkyne (a) or amine (b).

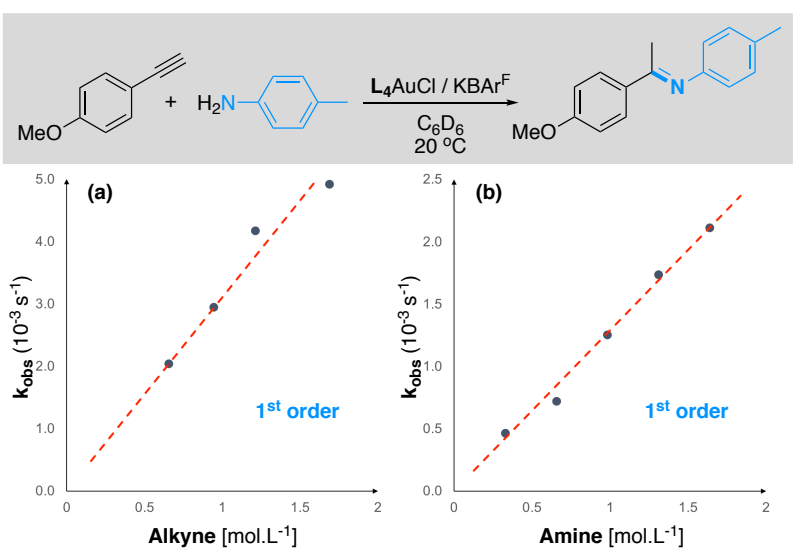

Figure 5. Rate dependence of the hydroamination on the concentration of alkyne (a) and amine (b).

this transformation. ${ }^{4}$ Upon comparing the catalytic activity of the CAAC complexes, we found that the smaller ${ }^{E t}$ CAAC-5 ( $\mathbf{L}_{\mathbf{1}}, \% \mathrm{~V}_{\text {bur }}$ : 43.2$)$ was deactivated by hydrazines in marked contrast with the bulkier $A d$ CAAC-5 ( $\mathbf{L}_{3}, \% \mathrm{~V}_{\text {bur }}$ : 48.4$)$ and ${ }^{E t}$ CAAC-6 $\left(\mathbf{L}_{2}, \% \mathrm{~V}_{\text {bur }}\right.$ : 47.4). We attribute this peculiar result to the propensity of $\mathbf{L}_{\mathbf{1}}$ to favor disproportionation to a catalytically inactive bis-ligated $\left(\mathbf{L}_{1}\right)_{2} \mathrm{Au}^{+}$in polar solvents. ${ }^{26} \mathrm{Apart}$ from $\mathbf{L}_{1}$, the order of activity $\mathbf{L}_{3}<\mathbf{L}_{2}<\mathbf{L}_{5}<\mathbf{L}_{\mathbf{4}}$ resembles that observed for the hydroamination, with the BiCAAC $\mathbf{L}_{\mathbf{4}}$ gold complex being the most active catalyst. As in the case of amines, we found that the catalytic activity is greatly impaired by strongly coordinating solvents (Figure 6c). Moreover, the early precipitation of the product prevented accurate reaction monitoring in benzene. Thus, using chloroform, we evaluated the concentration and the catalyst loading (Figure 6d). We selected $0.5 \mathrm{~mol} \%$ and $1.8 \mathrm{M}$ as a middle point between conversion and reaction time.

Reaction Scope: Using the optimized conditions obtained from our kinetic study $(0.5 \mathrm{~mol} \%$ of $\mathbf{L}_{4} \mathrm{AuCl} / \mathrm{KBAr}^{\mathrm{F}}$ in $\mathrm{CHCl}_{3}-1.8 \mathrm{M}$ ), we evaluated the scope of the reaction using a range of alkynes and 


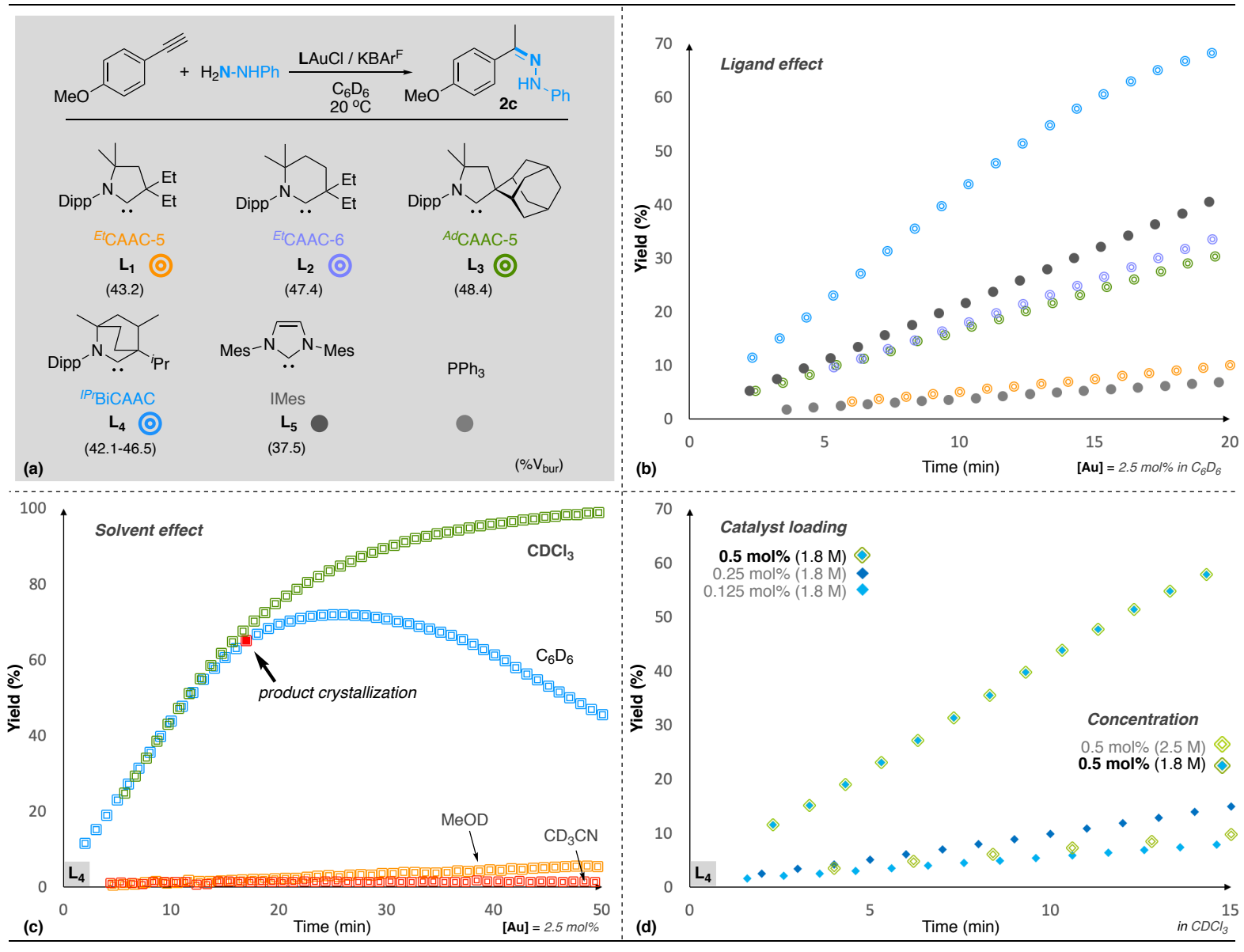

Figure 6. Optimization of the reaction conditions of the hydrohydrazination reaction using ${ }^{1} \mathrm{H}$ NMR kinetic monitoring

\section{Scheme 3. Substrate scope with hydrazines. NMR yields (isolated yields)}

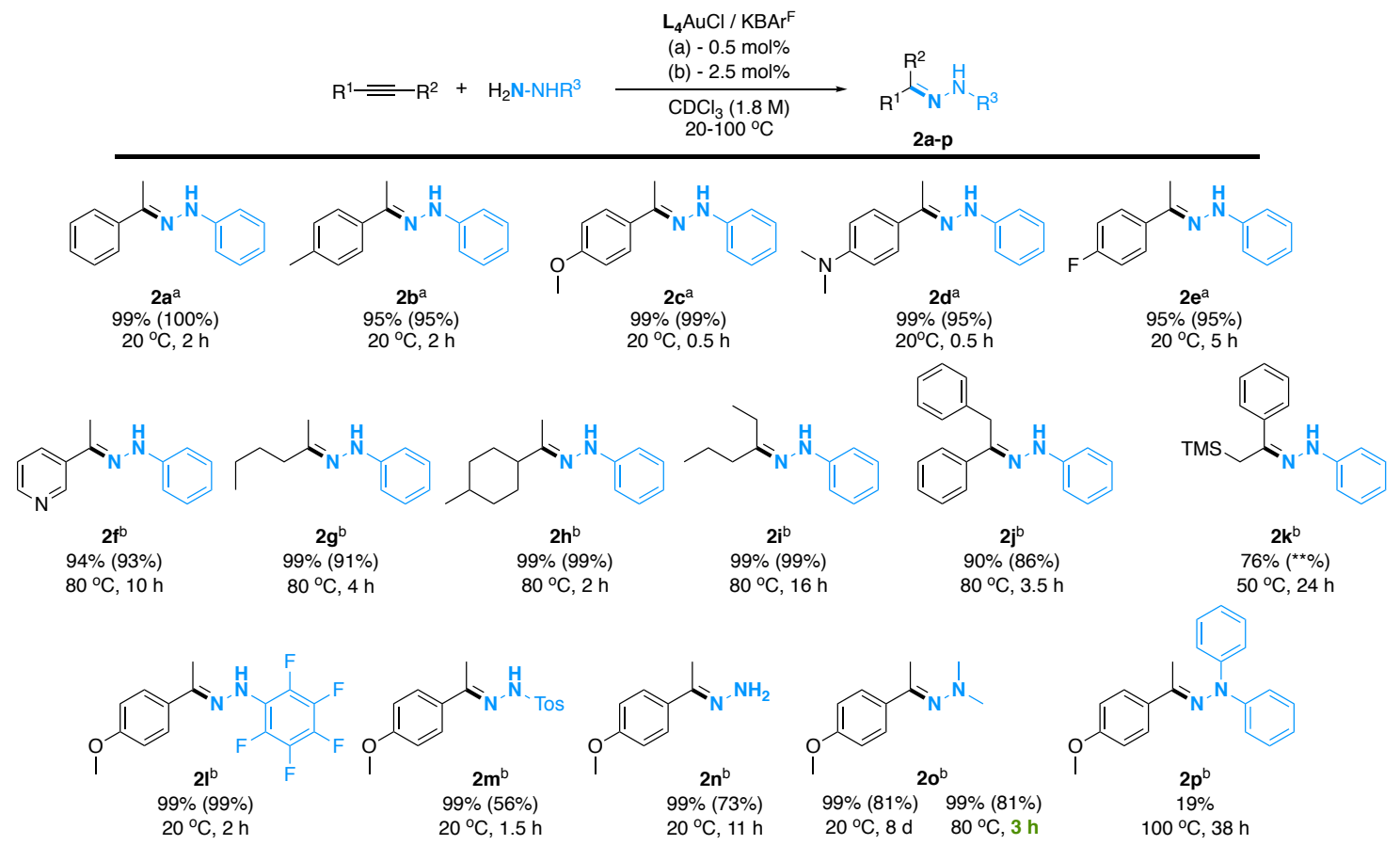


hydrazines (Scheme 3). With phenylhydrazine, we found this catalytic system to be very efficient with aryl substituted terminal alkynes at room temperature (2a-2e). With 3-ethynylpyridine (2f), however, the reaction was much slower, requiring $2.5 \mathrm{~mol} \%$ catalyst loading and $80{ }^{\circ} \mathrm{C}$ for $10 \mathrm{~h}$. Similarly, in the case of alkyl substituted terminal alkynes, as well as internal alkynes, higher reaction temperatures were required to reach high conversion within a reasonable timeframe (2g-2k). While both mono-substituted and parent hydrazine were readily converted under mild conditions (2l-2m), we found 1,1-disubstituted hydrazines (2027 and $\mathbf{2 p})$ to be much less reactive.

Hammett correlation study. A Hammett correlation study was performed to quantify the electronic influence of the substrates in the gold catalyzed hydrohydrazination of alkynes (Figure 7). Faster reaction kinetics were obtained for alkynes bearing electron donating phenyl para-substituents ( $\rho$ value $<0$ ), as observed in the hydroamination. The reverse is observed with phenyl-hydrazine, with decreasing rates obtained for electron donating substituents ( $\rho$ value $>0$ ). This observation is in good agreement with the experimental results (21-2o), and could be due to the higher binding affinity of the most basic hydrazines to the gold center. However, a computational study by Ujaque, Lledós et al. ${ }^{28}$ showed that the catalytic cycle of hydrohydrazination of alkynes does not depend on the energy barrier for the nucleophilic addition of the hydrazine on the Au- $\pi$ complex, but rather relies on hydrazine assisted intermolecular proton transfer. As a result, it is likely that the increased hydrazine $\mathrm{N}-\mathrm{H}$ bond acidity with electron-withdrawing phenyl substituents favors the reaction.
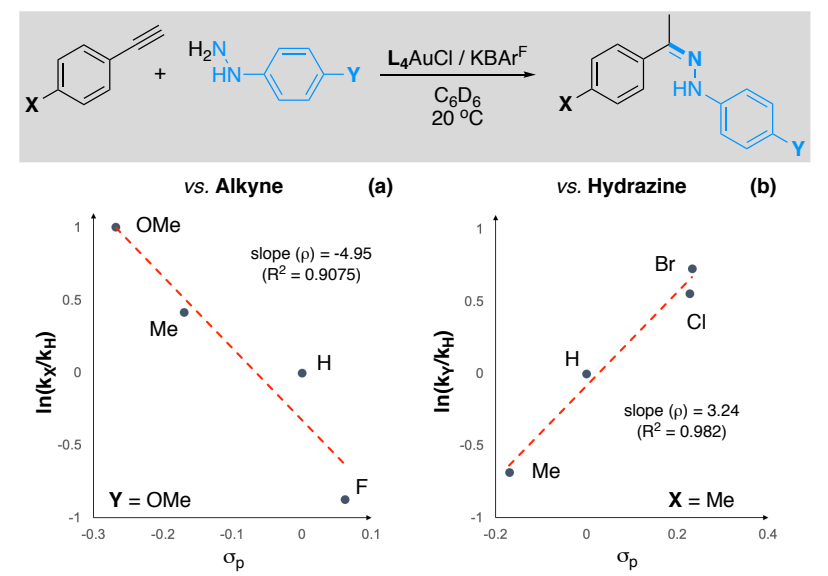

Figure 7 Hammett correlations of rates and substituents on either alkyne (a) or hydrazine (b).

Rate order. We established the rate order in the alkyne and the hydrazine under pseudo first-order conditions (excess of the spectator substrate; Figure 8). We found first order dependence for both reactants supporting that both alkyne and hydrazine are involved in the rate-limiting step. Together with the results obtained for the hydroamination, our data are in good agreement with both processes falling into the category of reactions involving nucleophilic attack of an amine on a gold activated carbon-carbon triple bond, followed by nucleophile assisted nitrogen to carbon atom proton transfer. ${ }^{4,28}$

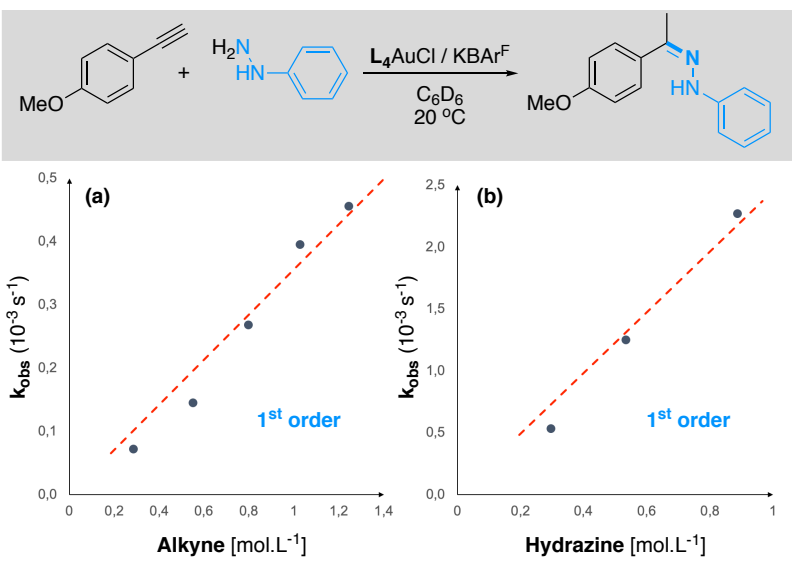

Figure 8. Rate dependence of the hydrohydrazination on the concentration of alkyne (a) and hydrazine (b).

\section{Rationalizing the ligand effect}

Catalyst stability. As already mentioned, low turnover numbers in gold catalysis often originate from catalyst degradation giving unreactive $\mathrm{Au}^{0}$ nanoparticles or unreactive $(\mathrm{L})_{2} \mathrm{Au}^{+}$species. $\mathrm{Xu}$ and coworkers disclosed that the decay of cationic gold $\left[\left(\mathrm{R}_{3} \mathrm{P}\right) \mathrm{Au}^{+}(\pi\right.$-alkyne)][OTf-] in solution is phosphine dependent. ${ }^{4}$ Buchwald-type biphenyl phosphine ligands were shown to afford very stable cationic gold complexes, 5,4 likely owing to secondary coordination to the distal phenyl substituent of the biphenyl unit. ${ }^{29}$ To estimate the propensity of CAAC ligands to stabilize cationic gold $(\mathrm{L}) \mathrm{Au}^{+}(\pi$-alkyne $)$ under pseudo-catalytic conditions, we performed a similar study. We examined the rate of decay at room temperature in cationic gold species bearing $\mathbf{L}_{\mathbf{1}}-\mathbf{L}_{\mathbf{4}}$ ligands, as well as the smaller ${ }^{M e}$ BiCAAC $\mathbf{L}_{6}$ and $\mathrm{Ph}_{3} \mathrm{P}$, in the presence of excess $n$ hexyne (Figure 9). As expected, we found ${ }^{E t} C A A C-5 \mathbf{L}_{\mathbf{1}}$, ${ }^{A d}$ CAAC-5 $\mathbf{L}_{3}$, and ${ }^{I P r}$ BiCAAC $\mathbf{L}_{\mathbf{4}}$ to yield very stable cationic gold species over a period of 4 days, whereas a rapid decay was observed with ${ }^{E t} \mathrm{CAAC}-6 \mathbf{L}_{2},{ }^{\mathrm{Me}} \mathrm{BiCAAC}$ $\mathbf{L}_{6}$ and $\mathrm{PPh}_{3}$. For $\mathbf{L}_{2}$ and $\mathrm{PPh}_{3}$, we attributed the decomposition to the formation of gold( 0 ) nanoparticles as highlighted by the observation of insoluble black deposits on the NMR sample. ${ }^{30}$ Because of the very different lifetime of $\left({ }^{I P r} \mathrm{BiCAAC}\right) \mathrm{AuCl}$ and ( $\left.{ }^{\mathrm{Me}} \mathrm{BiCAAC}\right) \mathrm{AuCl}$, we took a closer look at their behavior under catalytic conditions. As shown in Figure 10, in both hydroamination and hydrohydrazination, we 


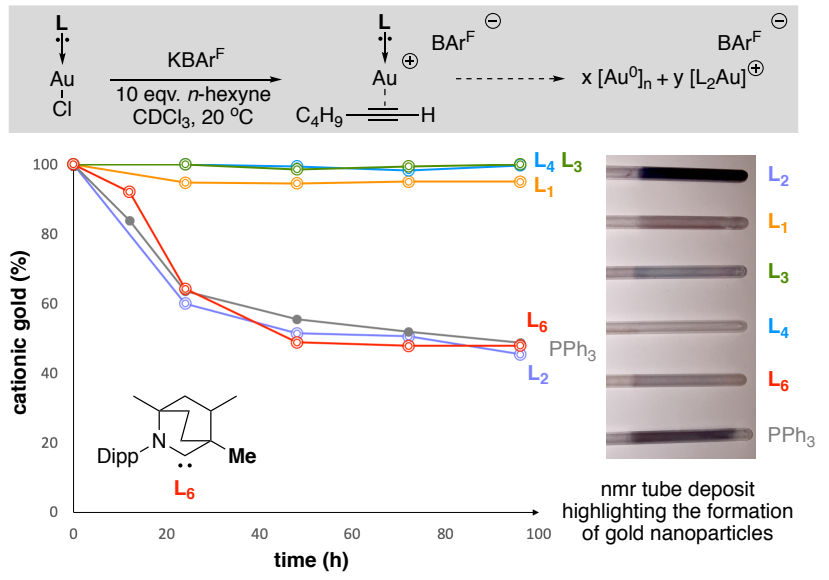

Figure 9. Ligand effects on the decay of [LAu(alkyne) $\left.{ }^{+}\right]\left[\mathrm{BAr}^{\mathrm{F}}{ }^{-}\right]$.

found that ( $\left.{ }^{I P r} \mathrm{BiCAAC}\right) \mathrm{AuCl}$ outperforms $\left({ }^{\mathrm{Me}} \mathrm{BiCAAC}\right) \mathrm{AuCl}$. We next attempted to isolate the putative intermediates of the catalytic cycle (Scheme 4). Complex $\left(\mathbf{L}_{4}\right) \mathrm{AuCl}$ reacted with a terminal or an internal alkyne affording stable $\left(\mathbf{L}_{4}\right) \mathrm{Au}^{+}$(alkyne) adducts with either $\mathrm{BF}_{4}$ or $\mathrm{BAr}^{\mathrm{F}}$ anions. One of these complexes was characterized by X-ray crystallography. In contrast, even with a large excess of alkyne, $\left(\mathbf{L}_{6}\right) \mathrm{Au}^{+}(\mathrm{al}-$ kyne) can only be characterized by NMR spectroscopy. After 2 days at room temperature, this complex disproportionates into the bis-ligated $\left(\mathbf{L}_{6}\right)_{2} \mathrm{Au}+$ (characterized by X-ray crystallography) and gold nanoparticles. Note that the predisposition of ${ }^{\mathrm{M} e}$ BiCAAC $\mathbf{L}_{\mathbf{6}}$ over ${ }_{I P r} \mathrm{BiCAAC} \mathbf{L}_{\mathbf{4}}$ to favor the formation of such bis-ligated complexes has already been observed with copper. ${ }^{12}$

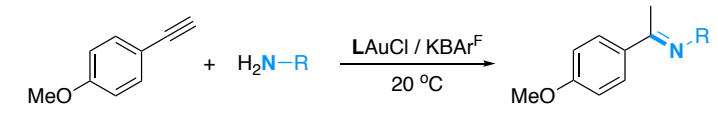

VS.

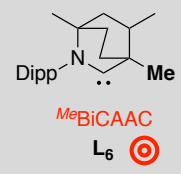

Hydroamination (a)
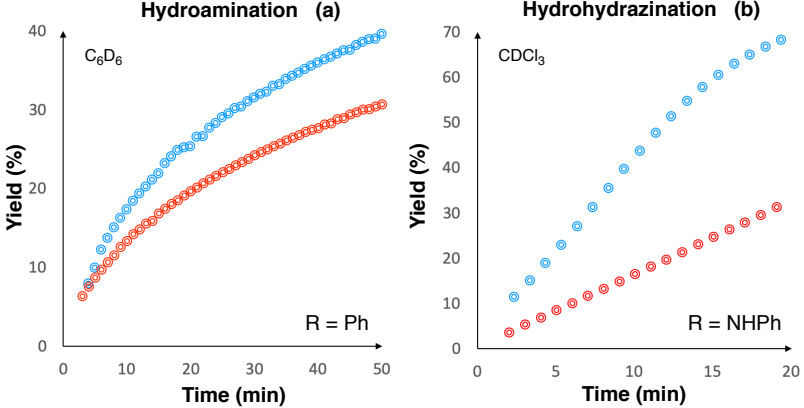

Figure 10. Comparison of the catalytic activity of $\mathbf{L}_{\mathbf{4}}$ and $\mathbf{L}_{6}$ in the hydroamination (a) and hydrohydrazination (b) reactions

Reaction of $\left(\mathbf{L}_{4}\right) \mathrm{Au}^{+}(\mathrm{TolCCH})$ with an excess of tolylamine or tosyl-hydrazine led to the expected formation of the corresponding imine $\mathbf{1 b}$ and hydrazone $\mathbf{2 q}$. More interestingly, these experiments also showed the concomitant formation of the Werner adducts $\left(\mathbf{L}_{4}\right) \mathrm{Au}^{+}\left(\mathrm{TolNH}_{2}\right)$ and $\left(\mathbf{L}_{4}\right) \mathrm{Au}^{+}\left(\mathrm{TosNHNH}_{2}\right)$ which further illustrates that ${ }^{I P r} \mathrm{BiCAAC} \mathbf{L}_{\mathbf{4}}$ is capable of stabilizing the gold center at every step of the catalytic cycle.

Scheme 4. Behavior of BiCAACAu+ toward alkynes, amines and hydrazines under stoichiometric conditions. For the X-ray structures of gold complexes, only the cations are shown.
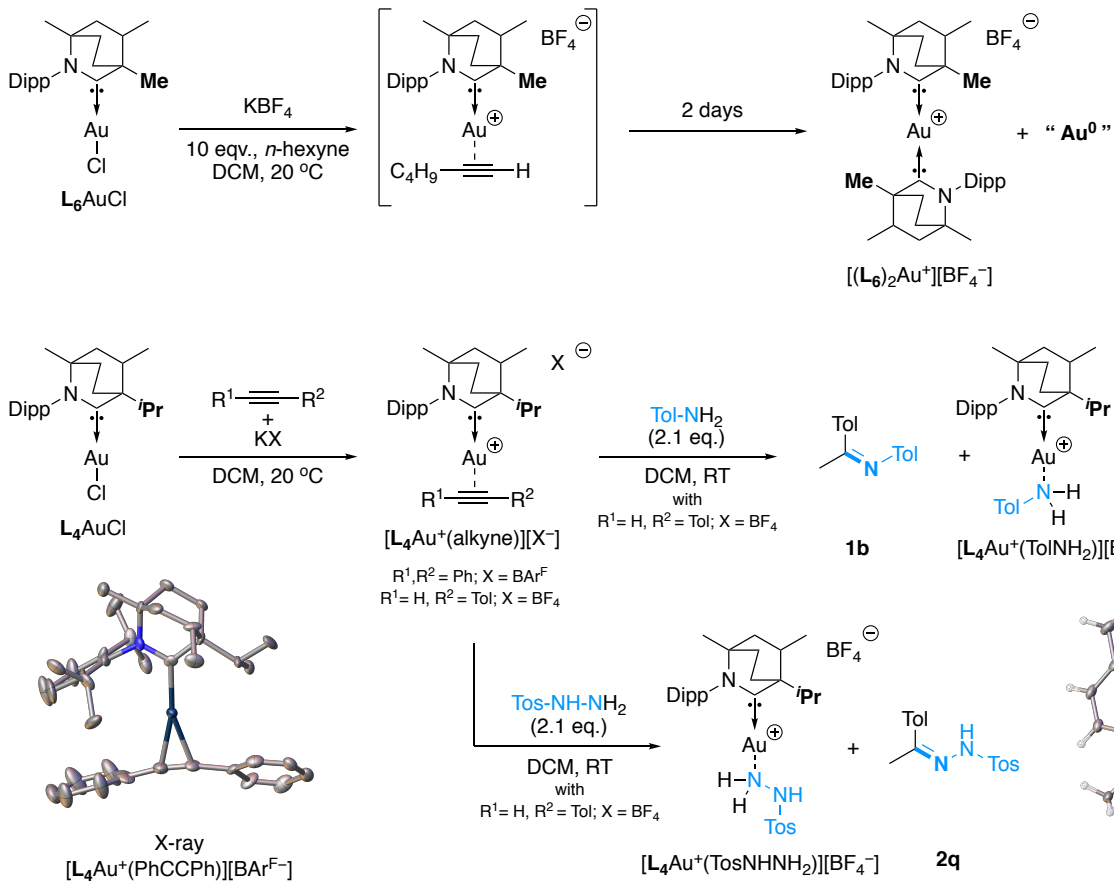

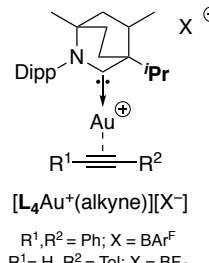

$\mathrm{R}^{1}, \mathrm{R}^{2}=\mathrm{Ph} ; \mathrm{X}=\mathrm{BArF}^{\mathrm{F}}$
$\mathrm{R}^{1}=\mathrm{H}, \mathrm{R}^{2}=\mathrm{Tol} ; \mathrm{X}=\mathrm{BF}_{4}$

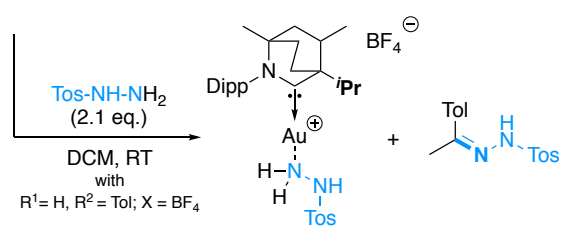

$\left[\mathbf{L}_{4} \mathrm{Au}^{+}\left(\mathrm{TosNHNH}_{2}\right)\right]\left[\mathrm{BF}_{4}^{-}\right]$
$2 q$
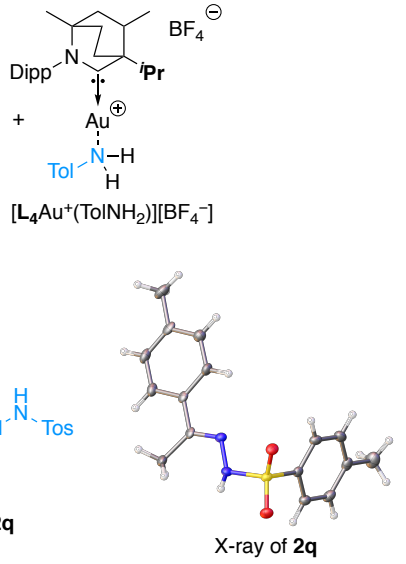
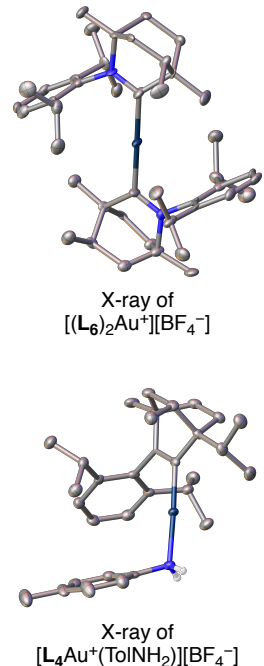

NB: solid state structure's anions were omited for clarity 
Rationalizing the ligand effects by DFT: All calculations were performed with the Gaussian09 program suite $^{31}$ using ultrafine ${ }^{32}(99,590)$ integration grids. ${ }^{33}$ All structures were optimized with the $\omega$ B97X-D ${ }^{34}$ functional and 6-31 $\mathrm{g}^{* * 35}$ basis set for all atoms except $\mathrm{Au}$, which was treated with the SDD ${ }^{36}$ basis set and ECP. Solvent was treated as a polarizable continuum of benzene. ${ }^{37}$ Ujaque, Lledós et al. computationally demonstrated that the energy of the transition state for substrate exchange between the Werner complex (catalyst resting state) and the active species $\left(\eta^{2}-\pi\right.$ complex) follows the order of stability of the $\pi$-complex, ${ }^{28}$ meaning that the more stable the $\pi$-complex, the lower the barrier for a given ligand. Using their work as a guiding principle we compared the energy differences between the Werner complexes of aniline and phenylhydrazine and the $\eta^{2}-\pi$ complex of phen ylacetylene for ( $\mathrm{L}) \mathrm{Au}^{+}$complexes bearing ligands $\mathbf{L}_{1}$ to $\mathbf{L}_{4}$ (Figure 11). Our DFT study clearly shows that in both cases the ${ }^{I P r}$ BiCAAC $\mathbf{L}_{\mathbf{4}}$ affords the most accessible

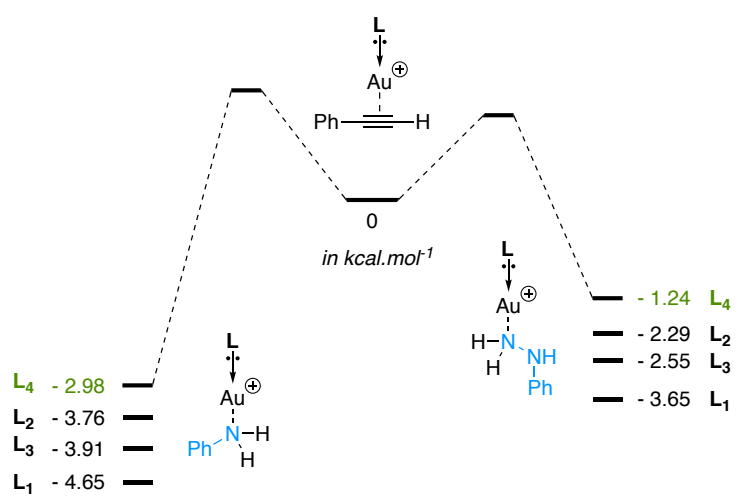

Figure 11. Energy profiles of substrate ligand exchanges as a function of $\mathrm{L}_{1}-\mathrm{L}_{4}$.

$\eta^{2}-\pi$ adducts. As highlighted previously, ${ }^{28}$ the barrier determining the rate of the catalytic cycle is not the barrier for the nucleophilic attack on the $\pi$-complex but rather the protonolysis of the migrated $\mathrm{Au}$-amide bond. ${ }^{38}$ In their report, Ujaque, Lledós et al. noted the trend of increasing barrier height for proton transfer with increasing donor-strength of the ligand (barrier height: CAAC > saNHC). This principle provides a conundrum for chemists designing catalysts, ${ }^{39}$ where there is a trade-off between the strength of the ligand to metal (L-M) bonds allowing for more durable catalysts and lower barriers to promote proton transfer ${ }^{40}$ Unarguably, the most ambiphilic ligands provide the strongest L-M bonds (i.e. $\mathbf{L}_{\mathbf{2}}>\mathbf{L}_{\mathbf{4}}>\mathbf{L}_{\mathbf{1}}=\mathbf{L}_{3}$ ) at the expense of an increased barrier for proton transfer. This could also explain why $\mathbf{L}_{2}$ performs much worse than several carbenes despite having the strongest M-L bond and a sterically flexible backbone. A good illustration of this statement is given by NHC $\mathbf{L}_{5}$ which performs surprisingly well compared to the more ambiphilic carbenes thanks to its relatively weak donor-ability resulting in a low barrier for proton transfer. Altogether, our data suggest that ${ }^{I P r}$ BiCAAC $\mathbf{L}_{\mathbf{4}}$ occupies the "sweet spot", featuring both a strong M-L bond and a relatively low barrier for proton transfer.

\section{Benchmarking ${ }^{I P r} \mathrm{BiCAAC} \mathrm{L}_{4}$ against state-of- the-art ligands}

The stability of gold complexes under prolonged reaction times and high temperatures is a prototypical limitation of most gold catalytic reactions. Capitalizing on the robustness of the $\mathbf{L}_{\mathbf{4}}$-Au bond, we envisaged that this complex should survive the experimental conditions used in the hydroamination of alkynes with the state-of-the-art phosphines $\mathbf{L}_{\mathbf{A}}$ and $\mathbf{L}_{\mathbf{B}}$, and NHC $\mathbf{L}_{\mathbf{C}}$ gold complexes, namely between 50 and $80^{\circ} \mathrm{C} .8,10 \mathrm{We}$ were pleased to witness a catalytic activity reaching nearly 17,000 TON in $19 \mathrm{~h}$ at $80{ }^{\circ} \mathrm{C}$ (Figure 12). We also evaluated the ( $\left.{ }^{I P r} \mathrm{BiCAAC}\right) \mathrm{AuCl}$ complex in the hydrohydrazination reaction, and obtained, under the same conditions, up to 27,000 TON.

\section{Conclusions}

In summary, we have reported a comprehensive study highlighting the reactivity of CAAC gold complexes in the hydroamination and hydrohydrazination of alkynes. Our kinetic measurements demonstrate that the ${ }^{I P r}$ BiCAAC $\mathbf{L}_{\mathbf{4}}$ provides a very active catalysts for these transformations, which is rationalized through mechanistic and DFT studies. We propose that the unique steric and electronic environment provided by the $\mathbf{L}_{\mathbf{4}}$ ligand singlehandedly shields the metal from degradation. Concomitantly it stabilizes the key $\pi$-alkyne complex active intermediate, and favors a low barrier for proton transfer. High TONs were obtained in the hydroamination and hydrohydrazination of alkynes, competing with recently reported, highly sophisticated phosphine and NHC complexes. Contrad-

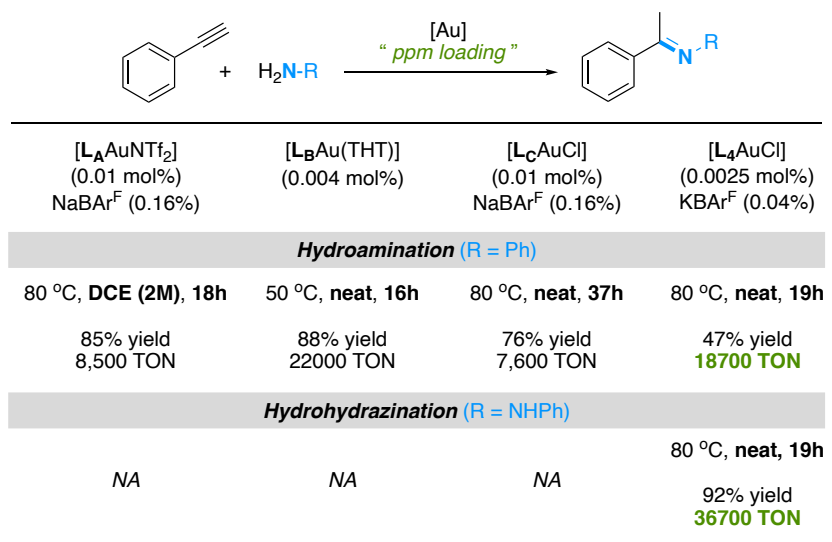

Figure 12. Benchmarking the catalytic activity of $I P r B i-$ CAAC gold chloride ( $\left.\mathrm{L}_{4} \mathrm{AuCl}\right)$ against state-of-the-art ligands. 
icting a general consensus that gold catalysts must be improved through the design of tailored secondary interactions, we have provided compelling evidence that there is still much to be gained in controlling the ligand core - that is, the metal-ligand bond itself. The remarkable progress made in the past 30 years in the design of stable carbenes have taught us that these species provide an exceptional handle for the tuning of the electronic and steric environment of a metal, including now the readily available BiCAAC ligands, ${ }^{12}$ one of the most recent additions to the CAAC family.

\section{Experimental Section}

All the catalytic experiments were performed in air using commercial grade solvents and reagents unless otherwise noted. All the kinetic experiments were performed three times to verify the reproducibility of the results. All other manipulations were performed using standard glovebox and Schlenk techniques. Glassware was dried in an oven overnight at $150^{\circ} \mathrm{C}$ or flame- dried before use. Benzene, diethyl ether, and n-pentane were freshly distilled over $\mathrm{Na}$ /benzophenone. Hexanes, dichloromethane, and chloroform were freshly distilled over $\mathrm{CaH}_{2}$. Deuterium-labeled solvents were purchased from Cambridge Isotope Laboratories. NMR: Multinuclear NMR data were recorded on a Varian INOVA 400 and 500 $\mathrm{MHz}$ or a Bruker Avance 300 and $600 \mathrm{MHz}$. Single-crystal $\mathrm{X}$-ray structure determinations were carried out at low temperature on a Bruker P4, Platform, or Kappa diffractometer equipped with a Mo $\left(\lambda=0.71073 \mathrm{~A}^{\mathrm{j}}\right)$ or $\mathrm{Cu}(\lambda=$ $1.54178 \mathrm{~A}^{\circ}$ ) radiation source and a Bruker APEX detector. Crystals were selected under oil, mounted on nylon loops, then immediately placed in a cold stream of nitrogen. All structures were solved by direct methods with SIR 2004 or SHELXS and refined by full-matrix leastsquares procedures utilizing SHELXL within the Olex 2 small-molecule solution, ${ }^{41}$ refinement, and analysis software package.

\section{General method for the hydroamination}

In air, a scintillation vial ( $3 \mathrm{~mL})$ was charged with IPrBiCAAC)AuCl/ KBArF (2.5 mol\%), C6H6 (1 mL) and the alkyne $(1.163 \mathrm{mmol}, 1.0$ eq.). After stirring for two minutes, the amine (1.163 mmol, 1.0 eq.) was added as a solution in C6H6 $(0.5 \mathrm{~mL})$. The mixture was then set stirring according to reaction time and temperature listed in scheme 3 . The products were purified by column chromatography using Florisil and eluting with a gradient mixture of DCM/hexane.

\section{General method for the hydrohydrazination}

In air, a scintillation vial ( $3 \mathrm{~mL})$ was charged with IPrBiCAAC)AuCl/ KBArF (0.5 mol\% for 2a-2e; $2.5 \mathrm{~mol} \%$ for $2 \mathrm{f}-$ 2p), C6H6 (200 $\mu \mathrm{L})$ and the alkyne $(0.357 \mathrm{mmol}, 1.0 \mathrm{eq}$.$) .$
After stirring for two minutes, the amine $(0.357 \mathrm{mmol}$, 1.0 eq.) was added as a solution in $\mathrm{C} 6 \mathrm{H} 6(200 \mu \mathrm{L})$. The mixture was then set to stir according to the reaction time and the temperature listed in scheme 4 . The products were purified by column chromatography using Florisil and eluting with a gradient mixture of DCM/hexane. NB: Air sensitive products were purified under argon using dried Florisil.

\section{ASSOCIATED CONTENT}

\section{Supporting information}

The Supporting Information is available free of charge at htpps://pubs.acs.org/doi/

Experimental procedures and characterization data, Xray crystallographic data and computational details.

\section{AUTHOR INFORMATION}

\section{Corresponding Author}

guybertrand@ucsd.edu

dbgrotjahn@sdsu.edu

rjazzar@ucsd.edu

\section{ORCID}

Sima Yazdani : 0000-0003-0261-7146

Glen P. Junor : 0000-0002-6733-3577

Jesse L. Peltier : 0000-0002-3493-2127

Rodolphe Jazzar : 0000-0002-4156-7826

Douglas B. Grotjahn : 0000-0002-2481-7889

Guy Bertrand : 0000-0003-2623-2363

\section{Funding Sources}

The authors declare no financial competing interests.

\section{ACKNOWLEDGMENT}

This work was supported at UCSD by the U.S. Department of Energy, Office of Science, Basic Energy Sciences, Catalysis Science Program, under Award No. DESC0009376 (GB). At SDSU, experimental work was supported by NSF award CHE-1800598 (D.B.G.), and by a SDSU University Graduate Fellowship (S.Y.). Thanks are due to the Alfred P. Sloan Foundations University Centre for Exemplary Mentoring (G.P.J.). This material is based upon work supported by the National Science Foundation Graduate Research Fellowship Program under Grant No. (DGE-1650112; G.P.J. and J. L. P.). Any opinions, findings, and conclusions or recommendations expressed in this material are those of the author(s) and do not necessarily reflect the views of the National Science Foundation. We also acknowledge the Keck Foundation for provided computational resources.

\section{REFERENCES}


1 Müller, T. E.; Hultzsch, K. C.; Yus, M.; Foubelo, F.; Tada, M. Hydroamination: direct addition of amines to alkenes and alkynes. Chem. Rev. 2008, 108, 3795-3892.

2 Mizushima, E.; Hayashi, T.; Tanaka, M. Au(I)-Catalyzed Highly Efficient Intermolecular Hydroamination of Alkynes. Org. Lett. 2003, 5, 3349-3352.

3 Selected reviews: (a) Widenhoefer, R. A.; Xiaoqing, H. Gold-catalyzed hydroamination of C-C multiple bonds. Eur. J. Org. Chem. 2006, 20, 4555-4563. (b) Hashmi, A. S. K. Gold-Catalyzed Organic Reactions. Chem. Rev. 2007, 107, 3180-3211. (c) Dorel, R.; Echavarren, A. M. Gold(I)-catalyzed activation of alkynes for the construction of molecular complexity. Chem. Rev. 2015, 115, 9028-9072. (d) Coman, S. M.; Parvulescu, V. I. Nonprecious Metals Catalyzing Hydroamination and C-N Coupling Reactions. Org. Process Res. Dev. 2015, 19, 1327-1355. (e) Asiri, A. M.; Hashmi, A. S. K. Gold-catalyzed reactions of diynes. Chem. Soc. Rev. 2016, 45, 4471-4503. (f) Wei, Y.; Shi, M. Divergent Synthesis of Carbo- and Heterocycles via Gold-Catalyzed Reactions. ACS Catal. 2016, 6, 2515-2524. (g) Alyabyev, S. B.; Beletskaya, I. P. Gold as a catalyst. Part I. Nucleophilic addition to the triple bond. Russ. Chem. Rev., 2017, 86, 689-749.

4 (a) Wang, W.; Hammond, G. B.; Xu, B. Ligand effects and ligand design in homogeneous Gold(I) catalysis. J. Am. Chem. Soc. 2012, 134, 5697-5705. (b) Malhotra, D.; Mashuta, M. S.; Hammond, G. B.; Xu, B. A Highly Efficient and Broadly Applicable Cationic Gold Catalyst. Angew. Chem., Int. Ed. 2014, 53, 4456-4459. (c) Lu, Z.; Hammond, G. B.; Xu, B. Improving Homogeneous Cationic Gold Catalysis through a MechanismBased Approach. Acc. Chem. Res. 2019, 52, 1275-1288.

5 Ligand effect in hydroamination: (a) Gorin, D. J.; Toste, F. D. Relativistic effects in homogeneous gold catalysis. Nature, 2007, 446, 395-403. (b) Gorin, D. J.; Sherry, B. D.; Toste, F. D. Ligand effects in homogeneous Au catalysis. Chem. Rev. 2008, 108, 3351-3378.

6 (a) Cheng, X.; Wang, Z.; Quintanilla, C. D.; Zhang, L. Chiral bifunctional phosphine ligand enabling gold-catalyzed asymmetric isomerization of alkyne to allene and asymmetric synthesis of 2,5- dihydrofuran. J. Am. Chem. Soc. 2019, 141, 3787-3791. (b) Liao, S.; Porta, A.; Cheng, X.; Ma, X.; Zanoni, G.; Zhang, L. Bifunctional ligand enables efficient gold-catalyzed hydroalkenylation of propargylic alcohol. Angew. Chem. Int. Ed. 2018, 57, 8250-8254. (c) Li, T.; Zhang, L. Bifunctional biphenyl-2ylphosphine ligand enables tandem gold-catalyzed propargylation of aldehyde and unexpected cycloisomerization. J. Am. Chem. Soc. 2018, 140, 17439- 17443. (d) Wang, Z.; Ying, A.; Fan, Z.; Hervieu, C.; Zhang, L. Tertiary amino group in cationic gold catalyst: tethered frustrated lewis pairs that enable ligand-controlled regiodivergent and stereoselective isomerizations of propargylic esters. ACS Catal. 2017, 7, 3676-3680. (e) Wang, Z.; Nicolini, C.; Hervieu, C.; Wong, Y.-F.; Zanoni, G.; Zhang, L. Remote cooperative group strategy enables ligands for accelerative asymmetric gold catalysis. J. Am. Chem. Soc. 2017, 139, 16064-16067. (f) Li, X.; Wang, Z.; Ma, X.; Liu, P.-N.; Zhang, L. Designed bifunctional phosphine ligand- enabled gold-catalyzed isomerizations of ynamides and allena- mides: stereoselective and regioselective formation of 1-amido-1,3-dienes. Org. Lett. 2017, 19, 5744-5747. (g) Li, X.; Liao, S.; Wang, Z.; Zhang, L. Ligand-accelerated gold-catalyzed addition of in situ generated hydrazoic acid to alkynes under neat conditions. Org. Lett. 2017, 19, 3687-3690. (h) Wang, Z.; Wang, Y.; Zhang, L. Soft propargylic deprotonation: designed ligand enables aucatalyzed isomerization of alkynes to 1,3-dienes. J. Am. Chem. Soc. 2014, 136, 8887-8890.

7 (a) Carreras, J.; Pereira, A.; Zanini, M. ; Echavarren, A. M. Variations on the theme of Johnphos gold(I) catalysts: arsine and carbene complexes with similar architectures.
Organometallics, 2018, 37, 3588-3597. (b) Zhang, J. Q.; Liu, Y.; Wang, X.-W.; Zhang, L. Synthesis of chiral bifunctional NHC ligands and survey of their utilities in asymmetric gold catalysis. Organometallics, 2019, 38, 3931-3938.

8 Wang, Y.; Wand, Z.; Li, Y.; Wu, G.; Gao, Z.; Zhang, L. “A general ligand design for gold catalysis allowing ligand-directed antinucleophilic attack of alkynes." Nat. Comm. 2014, 5, 3470.

9 Lavallo, V; Wright, J. H.; Tham, F. S.; Quinlivan, S. Perhalogenated Carba-closo-dodecaborate Anions as Ligand Substituents: Applications in Gold Catalysis. Angew. Chem. Int. Ed. 2013, 52, 3172-3176.

10 Tang, Y.; Benaissa, I.; Huynh, M.; Vendier, L.; Lugan, N.; Bastin, S.; Belmont, P.; César, V.; Michelet, V. An original L-shape, tunable N-heterocyclic carbene platform for efficient gold(i) catalysis. Angew. Chem. Int. Ed. 2019, 58, 7977-7981.

11 (a) Schießl, J.; Schulmeister, J.; Doppiu, A.; Wörner, E.; Rudolph, M.; Karch, R.; Hashmi, A. S. K., an industrial perspective on counter anions in gold catalysis: underestimated with respect to "ligand effects". Adv. Synth. Cat. 2018, 360, 2493-2502. (b) For ligand effects in gold catalysis operating over several bonds, see: Döpp, R.; Lothschuitz, C.; Wurm, T.; Pernpointner, M.; Keller, S.; Rominger, F.; Hashmi, A. S. K. Gold Catalysis: Hydrolysis of Di(alkoxy)carbenium Ion Intermediates as a Sensor for the Electronic Properties of Gold(I) Complexes. Organometallics 2011, 30, 5894- 5903.

12 Tomás-Mendivil, E. Hansmann, M. M., Weinstein, C., Jazzar, R., Melaimi, M, Bertrand, G. Bicyclic (alkyl)(amino)carbenes (BiCAACs): stable carbenes more ambiphilic than CAACs. J. Am. Chem. Soc. 2017, 139, 7753-7756.

13 (a) Lavallo, V.; Canac, Y.; Prasang, C.; Donnadieu, B.; Bertrand, G. Stable Cyclic (Alkyl)(Amino)Carbenes as Rigid or Flexible, Bulky, Electron-Rich Ligands for Transition-Metal Catalysts: A Quaternary Carbon Atom Makes the Difference. Angew. Chem. Int. Ed. 2005, 44, 5705-5709. (b) Soleilhavoup, M.; Bertrand, G. Cyclic (Alkyl)(Amino)Carbenes (CAACs): Stable Carbenes on the Rise. Acc. Chem. Res. 2015, 48, 256-266. (c) Melaimi, M., Jazzar, R., Soleilhavoup, M., Bertrand, G. "Cyclic(alkyl)(amino)carbenes (CAACs): Recent developments" Angew. Chem. Int. Ed. 2017, 56, 10046-1068. (d) Paul, U. S. D.; Radius, U. What Wanzlick Did Not Dare to Dream: Cyclic (Alkyl)(amino)carbenes (cAACs) as New Key Players in Transition-Metal Chemistry. Eur. J. Inorg. Chem. 2017, 3362-3375. (e) Kundu, S.; Sinhababu, S.; Chandrasekhar, V.; Roesky, H. W. Stable Cyclic (Alkyl)(Amino)Carbene (Caac) Radicals with Main Group Substituents. Chem. Sci. 2019, 10, 4727-4741.

14 Kinjo, R.; Donnadieu, B.; Bertrand, G. Gold-catalyzed hydroamination of alkynes and allenes with parent hydrazine. Angew. Chem. Int. Ed. 2011, 50, 5560-5563.

15 (a) Zeng, X.; Frey, G. D.; Kinjo, R.; Donnadieu, B.; Bertrand, G. Synthesis of a simplified version of stable bulky and rigid cyclic (alkyl)(amino)carbenes, and catalytic activity of the ensuing gold(i) complex in the three-component preparation of 1,2-dihydroquinoline derivatives. J. Am. Chem. Soc. 2009, 131, 86908696. (b) Zeng, X.; Soleilhavoup, M.; Bertrand, G. Gold-catalyzed intermolecular markovnikov hydroamination of allenes with secondary amines. Org. Lett. 2009, 11, 3166-3169.

16 Zeng, X.; Kinjo, R.; Donnadieu, B.; Bertrand, G. Serendipitous discovery of the catalytic hydroammoniumation and methylamination of alkynes. Angew. Chem. Int. Ed. 2010, 49, $942-$ 945.

17 Lavallo, V.; Frey, G. D.; Donnadieu, B.; Soleilhavoup, M.; Bertrand, G. Homogeneous catalytic hydroamination of alkynes and allenes with ammonia. Angew. Chem. Int. Ed. 2008, 47, 5224-5228. 
18 Weinstein, C. M., Junor, G. P. Tolentino, D. Jazzar, R., Melaimi, M. Bertrand, G. Highly ambiphilic room temperature stable sixmembered cyclic (alkyl)(amino)carbenes. J. Am. Chem. Soc. 2018, 140, 9255-9260.

19 Blackmond, D. G.; Kinetic Profiling of Catalytic Organic Reactions as a Mechanistic Tool. J. Am. Chem. Soc. 2015, 137, 1085210866.

20 (a) Sergievskaya, A. P.; Tatarchuk, V. V.; Makotchenko, E. V.; Mironov, I. V. Formation of Gold Nanoparticles During the Reduction of $\mathrm{HAuBr}_{4}$ In Reverse Micelles Of Oxyethylated Surfactant: Influence of Gold Precursor on the Growth Kinetics and Properties of the Particles. J. Mater. Res. 2015, 30, 1925-1933. (b) Yang, X.-F.; Wang, A.-Q.; Wang, Y.-L.; Zhang, T.; Li, J. Unusual Selectivity of Gold Catalysts for Hydrogenation of 1,3-Butadiene toward cis-2-Butene: A Joint Experimental and Theoretical Investigation. J. Phys. Chem. C 2010, 114, 3131-3139.

21 Klinkenberg, J. L.; Hartwig, J. F. Catalytic Organometallic Reactions of Ammonia. Angew. Chem. Int. Ed. 2011, 50, 86-95.

22 (a) Heaton, B. T.; Jacob, C.; Page, P. Transition Metal Complexes Containing Hydrazine and Substituted Hydrazines. Coord. Chem. Rev. 1996, 154, 193-229; (b) Van der Vlugt, J. I. Advances in selective activation and application of ammonia in homogeneous catalysis. Chem. Soc. Rev. 2010, 39, 2302-2322. (c) Young, P. C.; Green, S. L. J.; Rosair, G.; Lee, A.-L. Deactivation of gold (I) catalysts in the presence of thiols and amines characterization and catalysis. Dalton Trans. 2013, 42, 9645-9653. (d) Kim, J.; Kim, H. J.; Chang, S. Synthetic Uses of Ammonia in transition metal catalysis. Eur. J. Org. Chem. 2013, 3201-3213.

23 Manzano, R.; Wurm, T.; Rominger, F.; Hashmi, A. S. K. RoomTemperature Hydrohydrazination of Terminal Alkynes Catalyzed by Saturated Abnormal N-Heterocyclic Carbene-Gold(I) Complexes. Chem. Eur. J. 2014, 20, 6844-6848.

24 Martin, D.; Lassauque, N.; Donnadieu, B.; Bertrand, G. Cyclic Diaminocarbene with a Pyramidalized Nitrogen Atom: A Stable N-Heterocyclic Carbene with Enhanced Electrophilicity. Angew. Chem. Int. Ed. 2012, 51, 6172-6175.

25 López-Gómez, M. J.; Martin, D.; Bertrand, G. Anti-Bredt N-Heterocyclic Carbene: An Efficient Ligand for the Gold(I)-Catalyzed Hydroamination of Terminal Alkynes with Parent Hydrazine. Chem. Commun. 2013, 49, 4483-4485.

26 (a) Frey, G. D.; Dewhurst, R. D.; Kousar, S.; Donnadieu, B.; Bertrand, G., Cyclic (Alkyl)(amino)carbene Gold(I) Complexes: A Synthetic and Structural Investigation. J. Organomet. Chem. 2008, 693, 1674-1682. (b) Hamze, R.; Jazzar, R.; Soleilhavoup, M.; Djurovich, P. I.; Bertrand, G.; Thompson, M. E., Phosphorescent 2-, 3- and 4-Coordinate Cyclic (Alkyl)(amino)carbene (CAAC) $\mathrm{Cu}(\mathrm{I})$ Complexes. Chem. Commun. 2017, 53, 90089011.

27 A kinetic plot highlighting the difference in kinetic when performing the reaction at room temperature and at $80{ }^{\circ} \mathrm{C}$ is provided as supplementary material.

28 Couce-Rios, A.; Kovács, G.; Ujaque, G.; Lledós, A. Hydroamination of C-C Multiple Bonds with Hydrazine Catalyzed by N-Heterocyclic Carbene-Gold(I) Complexes: Substrate and Ligand Effects. ACS Catalysis 2015, 5, 815-829.

29 (a) Herrero-Goméz, E.; Nieto-Oberhuber, C.; Loṕez, S.; BenetBuchholz, J.; Echavarren, A. M. Cationic $\eta^{1} / \eta^{2}$-Gold(I) complexes of simple arenes. Angew. Chem. Int. Ed. 2006, 45, 54555459. (b) Li, Q.-S.; Wan, C.-Q.; Zou, R.-Y.; Xu, F.-B.; Song, H.-B.; Wan, X.-J.; Zhang, Z.-Z. Gold(I) $\eta^{2}$-arene complexes. Inorg. Chem. 2006, 45, 1888-1890. (c) Partyka, D. V.; Updegraff, J. B.; Zeller, M.; Hunter, A. D.; Gray, T. G. Probing the Steric Limits of Carbon-Gold Bond Formation: (Dialkylbiarylphosphine)gold(I) Aryls. Organometallics, 2009, 28, 1666-1674. (d) Touil, M.; Bechem, B.; Hashmi, A. S. K.; Engels, B.; Omary, M. A.; Rabaâ, H. Theoretical study of weak CC double bond coordination in a gold (I) catalyst precursor. J. Mol. Struc. THEOCHEM 2010, 957,
21-25. (e) Hashmi, A. S. K.; Bechem, B.; Loos, A.; Hamzic, M.; Rominger, F.; Rabaa, H. Gold Catalysis: Biarylphosphine Ligands as Key for the Synthesis of Dihydroisocoumarins. Aust. J. Chem. 2014, 67, 481-499.

30 The formation of such black deposits in the presence of reducing substrates in gold catalysis has been described early: (a) Hashmi, A. S. K.; Schwarz, L.; Choi, J.-H.; Frost, T. M. A New GoldCatalyzed C-C Bond FormationAngew. Chem., Int. Ed. 2000, 39, 2285-2288. (b) Hashmi, A. S. K.; Frost, T. M.; Bats, J. W. Highly Selective Gold-Catalyzed Arene Synthesis. J. Am. Chem. Soc. 2000, 122, 11553-11554.

31 Gaussian 09, Revision D.01, M. J. Frisch, G. W. Trucks, H. B. Schlegel, G. E. Scuseria, M. A. Robb, J. R. Cheeseman, G. Scalmani, V. Barone, B. Mennucci, G. A. Petersson, H. Nakatsuji, M. Caricato, X. Li, H. P. Hratchian, A. F. Izmaylov, J. Bloino, G. Zheng, J. L. Sonnenberg, M. Hada, M. Ehara, K. Toyota, R. Fukuda, J. Hasegawa, M. Ishida, T. Nakajima, Y. Honda, O. Kitao, H. Nakai, T. Vreven, J. A. Montgomery, Jr., J. E. Peralta, F. Ogliaro, M. Bearpark, J. J. Heyd, E. Brothers, K. N. Kudin, V. N. Staroverov, T. Keith, R. Kobayashi, J. Normand, K. Raghavachari, A. Rendell, J. C. Burant, S. S. Iyengar, J. Tomasi, M. Cossi, N. Rega, J. M. Millam, M. Klene, J. E. Knox, J. B. Cross, V. Bakken, C. Adamo, J. Jaramillo, R. Gomperts, R. E. Stratmann, O. Yazyev, A. J. Austin, R. Cammi, C. Pomelli, J. W. Ochterski, R. L. Martin, K. Morokuma, V. G. Zakrzewski, G. A. Voth, P. Salvador, J. J. Dannenberg, S. Dapprich, A. D. Daniels, O. Farkas, J. B. Foresman, J. V. Ortiz, J. Cioslowski, and D. J. Fox, Gaussian, Inc., Wallingford CT, 2013.

32 For a discussion about grid choice see: Bootsma, A. N.; Wheeler, $\mathrm{S}$. Popular integration grids can result in large errors in $\mathrm{dft}$ computed free energies. ChemRxiv 2019, DOI: 10.26434/chemrxiv.8864204.v5.

33 All computational results are available for download free of charge from UCSD Library Digital Collections. Yazdani, S.; Junor, G. P.; Pelier, J. L.; Jazzar, R.; Grotjahn, D. B.; Bertrand, G. (2020). Data from: Influence of the L Ligand $\left(\mathrm{R}_{3} \mathrm{P}, \mathrm{NHC}, \mathrm{CAAC}-\right.$ 5, CAAC-6, BiCAAC) on the Catalytic Activity of Gold Complexes in the Hydroamination and Hydrohydrazination of Alkynes. UC San Diego Library Digital Collections. Dataset. https://doi.org/10.6075/J0GB22FN

34 (a) Chai, J.-D.; Head-Gordon, M. Long-range corrected hybrid density functionals with damped atom-atom dispersion corrections. Phys. Chem. Chem. Phys. 2008, 10, 6615-6620. (b) For review on dispersion corrections: Grimme, S. Density functional theory with London dispersion corrections. Wiley Interdisciplinary Reviews: Computational Molecular Science 2011, 1, 211-228.

35 6-31g** basis set: Petersson, G. A.; Al-Laham, M. A. The complete basis set-quadratic configuration interaction family of methods. J. Chem. Phys. 1991, 94, 6081-6090.

36 (a) Schwerdtfeger, P.; Dolg, M.; Schwarz, W. H. E.; Bowmaker, G. A.; Boyd, P. D. W. Relativistic effects in gold chemistry. I. Diatomic gold compounds. J. Chem. Phys. 1989, 91, 1762-1774. (b) Andrae, D.; Haeussermann, U.; Dolg, M.; Stoll, H.; Preus, H. Energy-adjusted ab-initio pseudopotentials for the second and third row transition elements. Theor. Chem. Acc., 1990, 77, 123-141.

37 Tomasi, J.; Mennucci, B.; Cammi, R. Quantum Mechanical Continuum Solvation Models. Chem. Rev. 2005, 105, 2999-3094.

38 The critical influence of basicity on the protodeauration step has been demonstrated experimentally by Hashmi et al., see: (a) Hashmi, A. S. K.; Schuster, A. M.; Rominger, F. Gold Catalysis: Isolation of Vinylgold Complexes Derived from Alkynes. Angew. Chem. Int. Ed., 2009, 48, 8247-8249. (b) Hashmi, A. S. K.; Ramamurthi, T. D.; Rominger, F. On the Trapping of Vinylgold Intermediates. Adv. Synth. Catal. 2010, 352, 971-975. 
39 (a) Hashmi, A. S. K. Homogeneous Gold Catalysis Beyond Assumptions and Proposals-Characterized Intermediates. Angew. Chem. Int. Ed. 2010, 49, 5232-5241. (b) Ranieri, B.; Escofet, I.; Echavarren, A. M. Anatomy of gold catalysts: facts and myths. Org. Biomol. Chem. 2015, 13, 7103-7118.

40 Note that apart from these electronic effects, the catalyst lifetime has also been shown to be strongly influenced by sterics, see: (a) Jaimes, M. C. B.; Rominger, F.; Pereira, M. M.; Carrilho, R. M. B.; Carabineiro, S. A. C.; Hashmi, A. S. K. Highly active phosphite gold(i) catalysts for intramolecular hydroalkoxylation, enyne cyclization and furanyne cyclization. Chem. Commun. 2014, 50, 4937-4940. (b) Blanco Jaimes, M. C.; Bohling, C. R. N.; Serrano-Becerra, J. M.; Hashmi, A. S. K. Highly Active Mononuclear NAC-Gold(I) Catalysts. Angew. Chem. Int. Ed. 2013, 52, 7963-7966.

41 Dolomanov, O. V.; Bourhis, L. J.; Gildea, R. J.; Howard, J. A. K.; Puschmann, H. OLEX2: a complete structure solution, refinement and analysis program. J. Appl. Cryst. 2009, 42, 339-341.

\section{Table of Content Graphic}

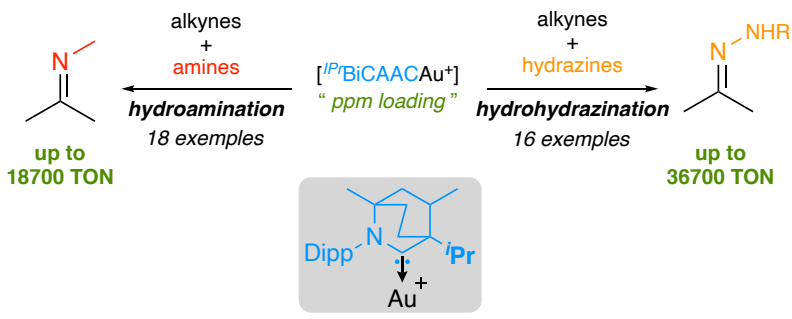

